\title{
Método Não-Linear de Pontos Interiores Aplicado à Minimização de Perdas em Sistemas de Potência ${ }^{1}$
}

L.V. BARBOZA² Universidade Católica de Pelotas, Rua Félix da Cunha 412, 96010000 Pelotas, RS, Brazil.

\begin{abstract}
Resumo. Este trabalho apresenta uma aplicação do método de Pontos Interiores não-linear ao problema da minimização de perda de potência ativa em linhas de transmissão de um sistema de energia elétrica. O problema de otimização é modelado como a minimização da geração de potência ativa nas barras de geração do sistema. Para uma demanda especificada no sistema elétrico, a diferença entre o somatório das potências ativas gerada e demandada corresponde à perda de potência na rede elétrica. Assim, minimizar a geração de potência ativa corresponde à minimização da perda de potência. Este problema de otimização é resolvido com a utilização do algoritmo de Pontos Interiores Primal-Dual, versão Preditor-Corretor. Para validar a metodologia proposta são apresentados resultados para sistemas teste do IEEE e para sistemas reais equivalentes da região Sul-Sudeste do Brasil. São mostrados resultados utilizando a metodologia convencional do fluxo de potência e os resultados obtidos com a aplicação da abordagem proposta.
\end{abstract}

\section{Introdução}

Nos dias atuais, a operação econômica, segura e confiável dos sistemas de energia elétrica passou a ser considerada como a principal meta a ser atingida pelas indústrias. O processo de privatização da rede elétrica brasileira, a partir do final do século do passado, tornou estas condições de operação dos sistemas de potência mais fundamentais ainda. Dentro deste novo paradigma, os processos de geração, transmissão e distribuição de energia elétrica devem ser realizados considerando-se novos parâmetros operacionais. Entre estes, devem ser ressaltados aqueles relacionados à operação econômica. A transmissão da energia elétrica tem que levar em conta a otimização dos equipamentos existentes na rede elétrica de modo a que se possa utilizá-los nos seus limites efetivos sem perda de confiabilidade. Este trabalho aborda um dos requisitos operacionais mais importantes para a utilização eficiente de uma rede de energia elétrica: a transmissão da energia elétrica com a menor perda de potência ativa nas linhas de transmissão. Com isso, se pode atender integralmente às demandas energéticas do sistema de potência com um mínimo de geração de energia. Associando-se a este objetivo uma minimização de custo

\footnotetext{
${ }^{1}$ Trabalho financiado através de convênio de P\&D entre a Companhia Estadual de Energia Elétrica (CEEE) e a Universidade Católica de Pelotas (UCPel).

${ }^{2}$ luciano@ucpel.tche.br
} 
de geração, pode-se ter um sistema de energia elétrica operando na condição mais econômica possível. O problema de fluxo de potência convencional $[9,16]$ utiliza uma metodologia de classificar o sistema elétrico por tipo de barras: $P V$ (barras de geração), $P Q$ (barras de carga) e folga (a barra de geração responsável pelo suprimento das perdas de potência). Nesta modelagem, a especificação da geração de potência ativa nas barras $P V$ é realizada através de casos históricos ou pela experiência dos operadores do sistema elétrico. Ao final do cálculo, toda a perda de potência do sistema tem que ser compensada pela geração de potência ativa na barra de folga. Por outro lado, a literatura apresenta algumas abordagens para o tratamento do problema da minimização da perda de potência ativa em linhas de transmissão. As metodologias sugeridas podem ser classificadas em: $(i)$ método da continuação [2] que consiste na execução repetida de vários problemas de fluxo de potência convencional ; (ii) programação linear [14]; (iii) análise de sensibilidade [6].

No presente estudo é proposta uma abordagem para o tratamento das perdas de potência ativa em linhas de transmissão como um problema de otimização restrita. O índice a ser otimizado corresponde à soma das gerações de potência ativa em todas as barras de geração. Para um sistema de potência, tem-se que a perda total de potência ativa corresponde à geração total de potência menos à soma de todas as demandas elétricas. Observe que, para uma determinada demanda total do sistema elétrico, se a geração total de potência for minimizada, isto implica em que as perdas de potência ativa também serão minimizadas. As restrições de igualdade correspondem aos balanços de potências ativa e reativa em todas as barras do sistema. As restrições de desigualdade modelam, por exemplo, limites operacionais e/ou de equipamentos constituintes do sistema elétrico. A solução do problema de otimização é realizada utilizando-se uma versão não-linear do método de Pontos Interiores Primal-Dual, versão Preditor-Corretor [11]. Esta versão do método tem sido muito aplicada a problemas de sistemas de potência $[13,15]$ pela facilidade com que as restrições de desigualdade são tratadas e pela eficiência do seu desempenho computacional, características essas que serão abordadas neste trabalho.

Este estudo está organizado da seguinte forma. Na seção 2 é apresentada uma revisão matemática do método de Pontos Interiores Primal-Dual na sua versão Preditor-Corretor. A seguir, introduz-se o problema da minimização da perda de potência ativa em linhas de transmissão em função das variáveis físicas envolvidas. Na seção 4 são mostrados resultados numéricos obtidos com a aplicação da metodologia proposta. Finalmente, conclusões e sugestões para trabalhos futuros são apresentadas.

\section{O Método de Pontos Interiores Primal-Dual, Versão Preditor-Corretor}

Considere o seguinte problema de otimização com restrições [3]

$$
\begin{array}{ll}
\text { Min } & f(x) \\
\text { s.a. } & g(x)=0 \\
& h(x) \geq 0,
\end{array}
$$


onde $x$ é o vetor das variáveis de otimização; $f(x)$ é a função objetivo a ser otimizada e $g(x)$ e $h(x)$ são funções não-lineares correspondentes, respectivamente, às restrições de igualdade e de desigualdade.

O tratamento das restrições de igualdade pelo método de Pontos Interiores é feito modificando-se a função objetivo. Estas restrições são incorporadas à função objetivo através dos multiplicadores de Lagrange. Por sua vez, as restrições de desigualdade são transformadas em igualdade pela adição de variáveis de folga nãonegativas $s$. Assim, o problema de otimização (2.1) pode ser reescrito como:

$$
\begin{array}{ll}
\text { Min } & f(x)-\lambda^{T} g(x) \\
\text { s.a. } & h(x)-s=0 \\
& s \geq 0,
\end{array}
$$

onde $\lambda$ corresponde ao vetor com os multiplicadores de Lagrange associados às restrições de igualdade.

O problema de otimização com restrições (2.2) pode ser transformado em um problema irrestrito utilizando-se o método de penalidades de Fiacco e McCormick [7], resultando em

$$
\operatorname{Min} f(x)-\lambda^{T} g(x)-\mu \sum_{i} \ln s_{i}-\pi^{T}[h(x)-s]
$$

onde $\pi$ é o vetor com os multiplicadores de Lagrange das restrições de desigualdade que foram transformadas em igualdade pela adição das variáveis de folga; o termo $\mu \sum_{i} \ln s_{i}$ é a função barreira logarítmica e garante a não-negatividade das variáveis de folga e $\mu$ é o parâmetro barreira do método de Pontos Interiores.

A função Lagrangeana correspondente ao problema de otimização (2.3) é

$$
£(x, s, \lambda, \pi)=f(x)-\mu \sum_{i} \ln s_{i}-\lambda^{T} g(x)-\pi^{T}[h(x)-s] .
$$

As condições de otimalidade de primeira ordem (condições de Karush-KuhnTucker) $[5,10]$ aplicadas à função Lagrangeana resultam em

$$
\begin{aligned}
\nabla_{x} £(x, s, \lambda, \pi) & =\nabla_{x} f(x)-G(x)^{T} \lambda-H(x)^{T} \pi=0 \\
\nabla_{s} £(x, s, \lambda, \pi) & =\mu e-S \pi=0 \\
\nabla_{\lambda} £(x, s, \lambda, \pi) & =-g(x)=0 \\
\nabla_{\pi} £(x, s, \lambda, \pi) & =-[h(x)-s]=0,
\end{aligned}
$$

onde $\nabla_{x} f(x)$ é o vetor gradiente da função objetivo; $G(x)$ e $H(x)$ são as matrizes Jacobianas das restrições de igualdade e de desigualdade, respectivamente, isto é,

$$
G(x)=\frac{\partial g(x)}{\partial x} \quad \text { e } \quad H(x)=\frac{\partial h(x)}{\partial x},
$$

$e$ corresponde a um vetor com 1's em todas as posições e $S$ é uma matriz diagonal com as variáveis de folga. 
Este sistema de equações não-lineares (2.5), pode ser resolvido pelo método de Newton-Raphson perturbado. Assim chamado pois, a cada iteração do processo, uma nova estimativa para o parâmetro barreira é calculada. Dessa forma, a cada iteração, deve-se resolver o seguinte sistema de equações lineares:

$$
\left[\begin{array}{cccc}
W(x, \lambda, \pi) & 0 & -G(x)^{T} & -H(x)^{T} \\
0 & -\Pi & 0 & -S \\
-G(x) & 0 & 0 & 0 \\
-H(x) & I & 0 & 0
\end{array}\right]\left[\begin{array}{c}
\Delta x \\
\Delta s \\
\Delta \lambda \\
\Delta \pi
\end{array}\right]=\left[\begin{array}{c}
-t \\
-(\mu e-S \pi) \\
g(x) \\
h(x)-s
\end{array}\right]
$$

onde $\Pi$ é uma matriz diagonal com os elementos do vetor $\pi$; $I$ é a matriz identidade; o vetor $t$ é definido como:

$$
t \equiv \nabla_{x} f(x)-G(x)^{T} \lambda-H(x)^{T} \pi
$$

e a matriz $W(x, \lambda, \pi)$ definida como:

$$
W(x, \lambda, \pi) \equiv \nabla_{x}^{2} f(x)-\sum_{i} \lambda_{i} \nabla_{x}^{2} g_{i}(x)-\sum_{j} \pi_{j} \nabla_{x}^{2} h_{j}(x)
$$

é a matriz Hessiana da função Lagrangeana em relação às variáveis de otimização.

Resolvida a equação matricial (2.7), obtém-se os incrementos nas variáveis primais e duais e a próxima etapa é determinar os comprimentos dos passos nos espaços primal e dual de modo a garantir a não-negatividade das variáveis de folga e os sinais adequados para os multiplicadores de Lagrange das restrições de desigualdade:

$$
\begin{gathered}
\gamma_{P}=\min \left\{\min _{\triangle s_{i}<0} \frac{s_{i}}{\left|\triangle s_{i}\right|}, 1\right\} \\
\gamma_{D}=\min \left\{\min _{\triangle \pi_{j}<0} \frac{\pi_{j}}{\left|\triangle \pi_{j}\right|}, 1\right\} .
\end{gathered}
$$

A nova aproximação para a solução é

$$
\begin{gathered}
x^{(k+1)}=x^{(k)}+\sigma \gamma_{P} \Delta x^{(k+1)} \\
s^{(k+1)}=s^{(k)}+\sigma \gamma_{P} \Delta s^{(k+1)} \\
\lambda^{(k+1)}=\lambda^{(k)}+\sigma \gamma_{D} \Delta \lambda^{(k+1)} \\
\pi^{(k+1)}=\pi^{(k)}+\sigma \gamma_{D} \Delta \pi^{(k+1)},
\end{gathered}
$$

onde $\sigma$ é uma constante que tem por finalidade garantir que as variáveis $s$ e $\pi$ não assumam valores iguais a zero [17]. A literatura recomenda, em termos de programação linear, a utilização de um valor igual a $0,9995$.

Finalmente, o método de penalidades de Fiacco e McCormick [7] estabelece que o parâmetro barreira $\mu$ deve diminuir durante o processo iterativo, tendendo a zero na convergência. A sua nova estimativa é

$$
\mu=\frac{s^{T} \pi}{2 n \beta}
$$

onde $\beta$ é um fator que controla o decrescimento do parâmetro barreira (recomendase utilizar um valor entre 10 e 20 [4]); $n$ é o número de restrições com limites e o 
numerador da equação (2.12) é conhecido como gap de complementariedade, o qual estima, a cada iteração, a distância entre as funções objetivo primal e dual para problemas de programação linear.

Esta solução do método de Pontos Interiores é conhecida como Primal-Dual.

Uma formulação alternativa deste método foi apresentada em [12] e reformulada em [11]. Esta ficou conhecida como método de Pontos Interiores Primal-Dual, versão Preditor-Corretor. A diferença fundamental entre as duas versões consiste em que, no Primal-Dual simplesmente aplica-se o método de Newton-Raphson para a obtenção dos pontos estacionários da função Lagrangeana, isto é, os pontos que satisfazem as condições de KKT, dadas por (2.5). Portanto, inicia-se um processo iterativo onde, a cada iteração, uma nova aproximação do ponto ótimo é determinada.

Na versão Preditor-Corretor, além de buscar, a cada iteração, uma melhor aproximação para a solução ótima, substitui-se esta nova aproximação diretamente nas condições de KKT. Com a aplicação desta metodologia [3], obtém-se o sistema de equações lineares para ser resolvido, a cada iteração, do processo iterativo:

$$
\left[\begin{array}{cccc}
W(x, \lambda, \pi) & 0 & -G(x)^{T} & -H(x)^{T} \\
0 & -\Pi & 0 & -S \\
-G(x) & 0 & 0 & 0 \\
-H(x) & I & 0 & 0
\end{array}\right]\left[\begin{array}{c}
\Delta x \\
\Delta s \\
\Delta \lambda \\
\Delta \pi
\end{array}\right]=\left[\begin{array}{c}
-t+z \\
-(\mu e-S \pi)+\Delta S \Delta \pi \\
g(x) \\
h(x)-s
\end{array}\right],
$$

onde $z=\left[\sum_{i} \lambda_{i} \nabla_{x}^{2} g_{i}(x)+\sum_{j} \pi_{j} \nabla_{x}^{2} h_{j}(x)\right] \triangle x$ e os demais termos são os mesmos da equação (2.7).

Observe que as matrizes de coeficientes dos sistemas de equações das formulações Primal-Dual, dada por (2.7), e Preditor-Corretor, dada por (2.13), são as mesmas. $\mathrm{Na}$ versão Preditor-Corretor, o vetor do lado direito do sistema linear apresenta termos não-lineares nos vetores $z$ e $\triangle S \triangle \pi$. Devido à presença destes termos, o sistema de equações (2.13) pode ser resolvido apenas de forma aproximada. Além disso, em termos de programação não-linear, o vetor $z$ pode ser desprezado na avaliação do vetor do lado direito do sistema linear [17], pois, em aplicações práticas, observa-se a sua pouca influência no processo iterativo.

Assim, em [11] é sugerido primeiramente se fazer uma etapa de predição na qual o problema original é resolvido, isto é, despreza-se a influência da barreira logarítmica. O sistema de equações lineares a ser resolvido é, portanto,

$$
\left[\begin{array}{cccc}
W(x, \lambda, \pi) & 0 & -G(x)^{T} & -H(x)^{T} \\
0 & -\Pi & 0 & -S \\
-G(x) & 0 & 0 & 0 \\
-H(x) & I & 0 & 0
\end{array}\right]\left[\begin{array}{c}
\Delta \hat{x} \\
\Delta \hat{s} \\
\Delta \hat{\lambda} \\
\Delta \hat{\pi}
\end{array}\right]=\left[\begin{array}{c}
-t \\
S \pi \\
g(x) \\
h(x)-s
\end{array}\right] .
$$

Com estes incrementos, o parâmetro barreira e os termos não-lineares podem ser estimados e o lado direito do sistema (2.13) pode ser determinado. Em [17] é sugerido pré-estimar-se o parâmetro barreira por:

$$
\mu=\left(\frac{\widetilde{\rho}}{\rho}\right)^{2}\left(\frac{\widetilde{\rho}}{2 n}\right),
$$


1. Inicialização das variáveis primais $(x$ e $s)$ e duais ( $\lambda$ e $\pi$ ) e do parâmetro barreira $\mu$.

2. Calcular o vetor gradiente da função Lagrangeana e calcular a sua norma infinita.

3. Testar as condições de convergência: tolerâncias para a norma infinita da função Lagrangeana e para o parâmetro barreira.

Critérios de convergência satisfeitos, solução determinada.

Senão, ir para o passo 4.

4. Calcular e fatorar a matriz Hessiana do sistema.

5. Etapa de predição: fazer $\mu=0$, recalcular o vetor do lado direito do sistema linear de equações e resolver o sistema de equações (2.14). Calcular os termos não-lineares e estimar dinamicamente o valor do parâmetro barreira $\mu$.

6. Etapa de correção: recalcular o vetor do lado direito do sistema linear de equações e resolver o sistema de equações (2.13). Os incrementos nas variáveis primais e duais são obtidos.

7. Determinar os comprimentos dos passos nos espaços primal e dual, $\gamma_{P}$ e $\gamma_{D}$.

8. Atualizar as variáveis primais e duais.

9. Estimar o novo valor do parâmetro barreira.

10. Retornar ao passo 2 .

Figura 1: Algoritmo de solução de um problema de otimização não-linear via método de pontos interiores primal-dual, versão preditor-corretor.

onde $\rho=s^{T} \pi$ corresponde ao gap de complementariedade sem atualização das variáveis; $\widetilde{\rho}=(s+\widetilde{\gamma} \triangle s)^{T}(\pi+\widetilde{\gamma} \triangle \pi)$ é o gap de complementariedade com atualização das variáveis e

$$
\widetilde{\gamma}=\min \left\{\min _{\triangle s_{i}<0} \frac{s_{i}}{\left|\triangle s_{i}\right|} \min _{\triangle \pi_{j}<0} \frac{\pi_{j}}{\left|\triangle \pi_{j}\right|}\right\} .
$$

Para obter a direção de busca da iteração corrente, realiza-se a etapa de correção. Nesta etapa, somente o vetor do lado direito do sistema linear é recalculado. Assim, resolve-se o sistema linear mostrado em (2.13). Para isso, necessita-se realizar apenas substituições diretas e inversas visto que a matriz do sistema linear já foi previamente fatorada na etapa de predição. Observe que a matriz de coeficientes do sistema linear da etapa de correção, equação (2.13), é a mesma da etapa de predição, equação (2.14).

Uma vez obtidos os incrementos nas variáveis primais e duais, a seqüência passa a ser a mesma do Primal-Dual. Determinam-se os comprimentos dos passos nos espaços primal e dual $\left(\gamma_{P}\right.$ e $\left.\gamma_{D}\right)$, atualizam-se as variáveis (vetores $\left.x, s, \lambda, \pi\right)$ e calcula-se o novo valor do parâmetro barreira $(\mu)$, dado por (2.12), válido para a próxima iteração.

A Figura 1 apresenta o algoritmo de solução de um problema de otimização não-linear utilizando o método de Pontos Interiores Primal-Dual, versão preditorcorretor. A etapa de inicialização das variáveis primais e duais deve ser realizada de modo a garantir a factibilidade destas. Por outro lado, para a inicialização do parâmetro barreira não existe uma regra que valha para qualquer problema de otimização. Esta deve ser feita de acordo com o problema físico modelado e o seu valor inicial pode variar entre $10^{-3}$ e $10^{2}$ [3]. A definição do valor inicial do parâmetro $\mu$, até o momento, consiste no principal problema numérico que afeta a convergência do processo iterativo dos métodos de pontos interiores. 


\section{O Problema da Minimização de Perda de Potên- cia Ativa}

Neste trabalho, o problema de minimização da perda de potência ativa em um sistema de energia elétrica é abordado através de um problema de minimização da potência ativa gerada. Observe que, para uma demanda especificada, a perda de potência ativa no sistema consiste na diferença entre os valores do somatório das potências geradas e o somatório das demandas especificadas. Os termos técnicos relativos à modelagem matemática do problema em questão podem ser encontrados em [9]. Dessa forma, o problema em análise pode ser modelado matematicamente como:

$$
\begin{aligned}
\operatorname{Min} & \sum_{i} P_{G_{i}} \\
\text { s.a. } & P_{j}^{\text {calc }}(x)-P_{G_{j}}+P_{d_{j}}=0 \\
& Q_{k}^{\text {calc }}(x)-Q_{G_{k}}+Q_{d_{k}}=0 \\
& x^{\text {min }} \leq x \leq x^{\max } \\
& Q_{G_{i}}^{\text {min }} \leq Q_{G_{i}}(x) \leq Q_{G_{i}}^{\max }
\end{aligned}
$$

onde $P_{G}$ é a potência ativa gerada, $\operatorname{com} i=1, \ldots, n p v$ (número de barras de geração); $P^{\text {calc }}$ corresponde à injeção de potência ativa calculada em função das variáveis de estado (termos não-lineares), com $j=1, \ldots, n b$ (número de barras do sistema

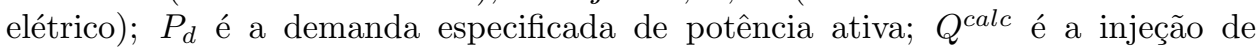
potência reativa calculada em função das variáveis de estado (termos não-lineares), com $k=1, \ldots, n p q$ (número de barras de carga); $Q_{G}$ é a potência reativa gerada (termos não-lineares), com $i=1, \ldots, n p v$ (número de barras de geração) e $Q_{d}$ é a demanda especificada de potência reativa.

O vetor das variáveis de estado, neste estudo, é composto por

$$
x=\left[\begin{array}{llll}
P_{G} & a & V & \delta
\end{array}\right]^{T},
$$

onde $P_{G}$ é o vetor com as potências ativas geradas; $a$ corresponde ao taps dos transformadores com comutação sob carga (LTC); $V$ é o vetor com as magnitudes das tensões complexas em todas as barras do sistema e $\delta$ é o vetor com os ângulos de fase das tensões complexas em todas as barras, exceto na barra de folga.

As variáveis $P_{G}, a$ e $V$ e a função $Q_{G}$ possuem limites mínimo e máximo que devem ser respeitados durante o processo de minimização.

As injeções de potências ativa e reativa são da forma

$$
\begin{aligned}
& P_{k}^{\text {calc }}(x)=G_{k k} V_{k}^{2}+V_{k} \sum_{m \in \Omega_{k}} V_{m}\left(G_{k m} \cos \delta_{k m}+B_{k m} \operatorname{sen} \delta_{k m}\right), \\
& Q_{k}^{\text {calc }}(x)=-B_{k k} V_{k}^{2}+V_{k} \sum_{m \in \Omega_{k}} V_{m}\left(G_{k m} \operatorname{sen} \delta_{k m}-B_{k m} \cos \delta_{k m}\right),
\end{aligned}
$$

onde $k$ é o número da barra em análise; $G$ e $B$ são matrizes com as partes real e imaginária da matriz admitância de barra $\left(Y_{\text {barra }}\right)$ e $\delta_{k m}$ corresponde à abertura angular da linha entre as barras $k$ e $m$, isto é, $\delta_{k m}=\delta_{k}-\delta_{m}$. 
A potência reativa gerada nas barras de geração pode ser expressa em função da injeção de potência reativa e da demanda especificada como:

$$
Q_{G_{i}}(x)=Q_{i}^{\text {calc }}(x)+Q_{d_{i}} .
$$

O problema de otimização enunciado por (3.1) é resolvido utilizando o método de Pontos Interiores Primal-Dual na versão Preditor-Corretor, conforme apresentado na seção 2. A metodologia proposta foi aplicada a vários sistemas-teste, tanto hipotéticos como realísticos. Os resultados e discussão estão apresentados na seção seguinte.

\section{Resultados Numéricos}

A metodologia apresentada na seção 3 foi implementada na linguagem Fortran ${ }^{\circledR}$ em um computador PC de $2.8 \mathrm{GHz}$, com $512 \mathrm{Mb}$ de memória RAM e com sistema operacional Windows. Para avaliar o seu desempenho, esta foi aplicada a vários sistemas-teste. Neste artigo, mostrar-se-á resultados para os sistemas-teste apresentados na Tabela 1. Nesta tabela, a coluna 2 refere-se ao número de barras do sistema elétrico, a coluna 3 , ao número de circuitos (linhas de transmissão e transformadores), a coluna 4, ao número de barras de geração, a coluna 5, ao número de transformadores LTC e a coluna 6, à dimensão do sistema linear de equações a ser resolvido, a cada iteração do processo iterativo.

Tabela 1: Principais características dos sistemas-teste.

\begin{tabular}{|l|c|c|c|c|c|}
\hline Sistema & $n b$ & $n c$ & $n p v$ & ntraf & dim \\
\hline IEEE & 57 & 80 & 7 & 15 & 586 \\
IEEE & 118 & 179 & 34 & 9 & 1.260 \\
SSB & 340 & 684 & 53 & 141 & 3.848 \\
SSB & 1.916 & 2.788 & 153 & 401 & 18.556 \\
\hline
\end{tabular}

Os dois primeiros sistemas são propostos pelo IEEE. Os outros dois são sistemas reais equivalentes da região Sul-Sudeste do Brasil (SSB).

Devido à dimensão dos sistemas de equações lineares ser muito elevada, sistemas (2.13) e (2.14), recomenda-se adotar um método de fatoração para a matriz de coeficientes robusto do ponto de vista numérico. Neste trabalho, optou-se por utilizar a rotina MA27AD da Harwell [1] que, entre outras vantagens, permite a fatoração simbólica da matriz de coeficientes e, durante o processo iterativo, apenas os valores numéricos da fatoração são atualizados.

O desempenho da metodologia foi avaliado tanto em termos matemático-computacionais como também na qualidade das soluções fornecidas. As soluções obtidas nos testes foram comparadas com as soluções determinadas através das técnicas convencionais de fluxo de carga, muito utilizadas pelas indústrias de energia elétrica. A modelagem matemática do problema não-linear de fluxo de carga pode ser encontrada em [16]. 
Os testes realizados consideraram uma tolerânicia de $10^{-3}$ para a norma infinita da função Lagrangeana e de $5 \cdot 10^{-5}$ para o valor do parâmetro barreira $\mu$. A inicialização das variáveis primais foi realizada com base no ponto médio do seu intervalo de variação, as variáveis duais foram inicializadas com valores 1 e o valor inicial do parâmetro barreira foi especificado em $10^{-3}$.

A Tabela 2 apresenta os resultados obtidos para os processos iterativos. Nesta tabela, a coluna $2(F P C)$ refere-se aos resultados do fluxo de potência convencional; a coluna 3 , aos resultados obtidos com a abordagem proposta - mínima perda otimizada $(M P O)$ e a coluna 4 , à demanda de potência ativa no sistema elétrico. Nas colunas do $F P C$ e do $M P O$, são mostrados o número de iterações e o tempo computacional (em segundos) requeridos pelo processo iterativo. O programa computacional utilizado para o FPC foi implementado pelo autor deste trabalho baseado em [16].

Tabela 2: Resultados dos processos iterativos.

\begin{tabular}{|l|c|c|c|c|c|}
\hline Sistema & \multicolumn{2}{|c|}{$F P C$} & \multicolumn{2}{|c|}{$M P O$} & Demanda Especificada (MW) \\
\hline IEEE-57 & 3 & 0,002 & 8 & 0,078 & $1.250,80$ \\
IEEE-118 & 3 & 0,005 & 8 & 0,147 & $4.125,00$ \\
SSB-340 & 6 & 0,031 & 19 & 0,821 & $33.077,83$ \\
SSB-1916 & 7 & 0,406 & 45 & 10,912 & $35.038,30$ \\
\hline
\end{tabular}

Pela análise da Tabela 2, percebe-se que, conforme esperado, o processo de otimização exige um número maior de iterações para a convergência e, por conseguinte, um tempo computacional maior. Nesta modelagem, em especial, o aumento no número de iterações para o processo de otimização deve-se, em parte, ao fato de que está se utilizando como variáveis de otimização as variáveis $V$ e $a$. Sabe-se, da teoria de sistemas de energia elétrica, que estas variáveis possuem um alto grau de acoplamento. Dessa forma, modificação nos valores de uma delas, afeta por de forma significativa os valores da outra.

A Tabela 3 apresenta os resultados obtidos do ponto de vista de qualidade de solução oferecida pelas metodologias. A coluna 2 mostra a perda de potência total no sistema elétrico quando empregada a abordagem convencional do fluxo de potência (em MW); a coluna 3, a perda (em MW) quando utilizada a abordagem de otimização proposta neste trabalho. Os níveis de demanda especificados para os sistemas são aqueles apresentados na Tabela 2.

Tabela 3: Qualidade das soluções - Perda total de potência ativa.

\begin{tabular}{|l|c|c|}
\hline Sistema & $F P C$ & $M P O$ \\
\hline IEEE-57 & 61,80 & 23,18 \\
IEEE-118 & 136,65 & 46,99 \\
SSB-340 & $1.023,57$ & 785,82 \\
SSB-1916 & $1.749,13$ & $1.406,58$ \\
\hline
\end{tabular}


Analisando a Tabela 3, percebe-se que a aplicação da metodologia proposta oferece, do ponto de vista econômico de operação do sistema de energia elétrica, uma solução de melhor qualidade. Pode-se determinar a partir desta tabela que a perda de potência ativa total nos sistemas eléticos estudados reduz-se em $62 \%$ para o sistema IEEE-57, em 65\% para o IEEE-118, em 23\% para o SSB-340 e em 20\% para o SSB-1916.

\section{Conclusões}

Este trabalho apresentou uma metodologia para o tratamento das perdas de potência ativa em redes de energia elétrica. A abordagem proposta modelou o processo físico na forma de um problema de otimização.

As principais conclusões que se pode inferir a partir dos resultados obtidos são:

- a perda de potência ativa em sistemas elétricos de potência pode ser modelada através de um problema de otimização com restrições;

- a solução deste problema pode ser realizada com a utilização do método nãolinear de Pontos Interiores Primal-Dual, na sua versão Preditor-Corretor;

- o processo de otimização, como era esperado, exige um número maior de iterações e um esforço computacional maior para a convergência do processo iterativo em comparação com o fluxo de carga convencional, aumentando, por conseguinte, o seu tempo computacional;

- a qualidade da solução fornecida pela técnica proposta é melhor do que aquela fornecida pelas técnicas tradicionais do fluxo de carga convencional, ou seja, com a utilização da abordagem proposta é possível reduzir a perda de potência ativa no sistema elétrico;

- a ferramenta matemático-computacional implementada pode ser de grande utilidade para as indústrias de energia elétrica para os estudos de planejamento de operação e expansão da rede elétrica.

Como futuros trabalhos, sugere-se o estudo de metodologias que visem a reduzir o elevado número de iterações do processo iterativo quando da aplicação da metodologia proposta a sistemas elétricos realísticos de grande porte e, por conseguinte, diminuir o esforço computacional necessário para a obtenção da solução, diminuindo o tempo exigido para o processo iterativo.

\footnotetext{
Abstract. This work presents an application of the nonlinear Interior Point method to the active power loss minimization problem in electric power systems. The optimization problem is modeled as the active power generation minimization at all generation buses. For a specified demand in the electric network, the difference between the summations of the generated active power and the specified active power demand corresponds to the total active power loss in the system. Thus, minimizing the total active power generation corresponds to the minimization of the total loss. This problem is solved using the nonlinear Predictor-Corretor
} 
Primal-Dual Interior Point method. In order to assess the behavior of the proposed approach, numerical results are presented for a large range of test-systems, including IEEE test-systems and equivalent real test-systems from the South-Southeastern region of Brazil. The results compare the conventional methodology used in power electric systems (load flow) to those obtained by using the proposed optimization technique.

\section{Referências}

[1] Computational Science \& Engineering Department, Harwell subroutine library, 2004. (Disponível em http://www.cse.clrc.ac.uk/nag/hsl/).

[2] D.A. Alves, L.C.P. Silva, C.A. Castro, V.F. Costa, Continuation load flow method parameterized by transmission line power losses, in "PowerCon 2000", pp. 763-768, Perth, 2000.

[3] L.V. Barboza, "Análise do Máximo Carregamento de Sistemas de Potência via Métodos de Pontos Interiores", Dissertação de Mestrado, PPGEE, UFSC, Florianópolis, SC, 1997.

[4] L.V. Barboza, "Análise e Desenvolvimento de Metodologias Corretivas para a Restauração da Solução das Equações da Rede Elétrica", Tese de Doutorado, PPGEE, UFSC, Florianópolis, SC, 2001.

[5] M.S. Bazaraa, C.M. Shetty, "Nonlinear Programming: Theory and Algorithms", John Wiley \& Sons, New York, 1979.

[6] M. El Arini, Effects of voltage sensitive loads on optimum capacitor allocation for power system, in "Melecon'96", pp. 1598-1602, Bari, 1996.

[7] A.V. Fiacco, G.P. McCormick, "Nonlinear Programming: Sequential Unconstrained Minimization Techniques", John Wiley \& Sons, New York, 1968.

[8] F. Glimn, G.W. Stagg, Automatic calculation of load flows, AIEE Transactions on Power Apparatus and Systems, 76 (1957), 817-828.

[9] J.D. Glover, M. Sarma, "Power System Analysis and Design", Brooks/Cole, Pacific Grove, USA, 2002.

[10] D.M. Greig, "Optimisation", Longman, London, 1980.

[11] S. Mehrotra, On the implementation of a primal-dual interior point method, SIAM Journal on Optimization, 2 (1992), 575-601.

[12] S. Mizuno, M. J. Todd, Y. Ye, Anticipated behaviour of the path following algorithms for linear programming, Technical report 878, School of Operations Research and Industrial Engineering, Cornell University, 1989.

[13] J.A. Momoh, M.E. Hawary, R. Adapa, A review of selected optimal power flow literature to 1993 - Newton linear programming and Interior Point methods, IEEE Transactions on Power Systems, 14, No. 1 (1999), 105-111. 
[14] M. Olofsson, G. Andersson, L. Soder, Linear programming based optimal power flow using second order sensitivities, IEEE Transactions on Power Systems, 3 (1995), 1691-1697.

[15] V.H. Quintana, G.L.T. Torres, J. Medina-Palomo, Interior Point methods and their applications to power systems: A classification of publications and software codes, IEEE Transactions on Power Systems, 15, No. 1 (2000), 170-176.

[16] G.W. Stagg, A.H. El-Abiad, "Computer Methods in Power System Analysis", McGraw-Hill, Tokyo, 1968.

[17] Y.C. Wu, A.S. Debs, R.E. Marsten, A direct nonlinear predictor corrector primal dual interior point algorithm for optimal power flows, IEEE Transactions on Power Systems, 9, No. 1 (1994), 876-883. 